\title{
Self-reported alteration of sense of smell or taste in patients with COVID-19: a systematic review and meta-analysis on 3563 patients*
}

\author{
Daniele Borsetto', Claire Hopkins', Veronica Philips², Rupert Obholzer' ${ }^{1}$ \\ Giancarlo Tirelli, Jerry Polesel ${ }^{4}$, Paolo Boscolo-Rizzo ${ }^{5}$ \\ 'Department of Otolaryngology, Guy's and St. Thomas' Hospital NHS Foundation Trust, London, United Kingdom \\ ${ }^{2}$ University of Cambridge Medical Library, Cambridge, United Kingdom \\ ${ }^{3}$ Section of Otorhinolaryngology, Azienda Sanitaria Universitaria Integrata di Trieste, Trieste, Italy \\ ${ }^{4}$ Centro di Riferimento Oncologico di Aviano (CRO) IRCCS, Aviano, Italy \\ Department of Neurosciences, Section of Otorhinolaryngology, University of Padova, Treviso, Italy
}

Rhinology 58: 5, 430 - 436, 2020

https://doi.org/10.4193/Rhin20.185

*Received for publication:

April 22, 2020

Accepted: May 13, 2020

\begin{abstract}
Background: Emerging reports suggest that new onset of smell or taste loss are potential early clinical markers of SARS-CoV-2 infection, but it remains unclear as to what extent. Therefore, the purpose of this study is to systematically assess the prevalence of self-reported altered sense of smell or taste in patients with confirmed SARS-CoV-2 infection, overcoming the limitations of individual studies by meta-analysis of pooled data.
\end{abstract}

Methods: The databases Medline, Embase, Web of Science, Scopus and MedRxiv's set were searched from inception to the 4th May 2020. This study was conducted following the PRISMA checklist.

Results: 18 studies met the eligibility criteria out of the 171 initially screened citations. The overall prevalence of alteration of the sense of smell or taste was $47 \%$, but estimates were $31 \%$ and $67 \%$ in severe and mild-to-moderate symptomatic patients, respectively. The loss of smell and taste preceded other symptoms in $20 \%$ of cases and it was concomitant in $28 \%$.

Conclusions: Based on this meta-analysis, we recommend self-isolation and testing, where possible, for patients complaining smell or taste impairment during COVID-19 pandemic in order to prevent spread of disease and propose the inclusion of loss of smell and taste as recognized symptoms of SARS-CoV-2 in the World Health Organization and other relevant regulatory body's lists.

Key words: SARS-CoV-2, Covid-19, anosmia, smell loss, ageusia, taste loss

\section{Introduction}

The COVID-19 pandemic, driven by the SARS-CoV-2 novel coronavirus infection, has spread globally since December 2019. The typical spectrum of clinical presentation of COVID-19 has been reported as ranging from asymptomatic infection to severe respiratory failure, with the main symptoms being fever, dry and productive cough, fatigue, myalgia, and dyspnoea ${ }^{(1)}$.

Previous strains of coronavirus have showed central nervous system involvement, propagating from within the olfactory bulb, through the olfactory neuroepithelium (2). More recently, it was observed that that olfactory sustentacular cells that maintain the integrity of olfactory sensory neurons, but not olfactory sensory neurons, co-express the transmembrane serine protease 2 and angiotensin-converting enzyme 2 (ACE2), the two key players in the binding and entry of the SARS-CoV-2 into human cells ${ }^{(3)}$. Consequently, altered sense of smell or taste have been first anecdotally and then more robustly reported in COVID-19 patients across Europe and Asia ${ }^{(4-6)}$.

Early, largely anecdotal, reports suggest that new onset of smell or taste loss are potential early symptoms or subclinical markers of SARS-CoV-2 infection, but it remains unclear to what extent. The identification of a comprehensive spectrum of symptoms 
associated to SARS-CoV-2 infection is of clinical interest to identify infected patients for isolation as early as possible where access to testing is restricted, or to facilitate early targeted testing where possible. For this purpose, it is of primary importance to define symptoms that patients can identify by themselves, without needing to visit healthcare professionals to perform formal olfactory testing, which is not widely available outside of research settings or highly specialized clinics. Therefore, the purpose of this meta-analysis is to systematically assess the prevalence of self-reported altered sense of smell or taste in patients with confirmed SARS-CoV-2 infection, overcoming the limitations of individual studies by meta-analysis of pooled data.

\section{Materials and methods}

\section{Search strategy}

This study was conducted following the preferred reporting items for systematic reviews and meta-analyses (PRISMA) checklist ${ }^{(7)}$. The search strategy was developed and conducted by a medical librarian (VP). Prior to conducting the searches, the search terms were peer reviewed by another medical librarian according to PRESS criteria. (https://www.sciencedirect.com/ science/article/pii/S0895435616000585).

The databases Medline (via Ovid), Embase (via Ovid), Web of Science (core collection) and Scopus were searched from inception to the 4th May 2020 with variants of the following search terms, which were in the title, abstract and keyword fields, as well as in MeSH term field when these existed in the database.

(( ("Corona virinae" or "corona virus" or Coronavirinae or coronavirus or COVID or nCoV) adj4 ("19" or "2019" or novel or new)) or (("Corona virinae" or "corona virus" or Coronavirinae or coronavirus or COVID or nCoV) and (wuhan or china or chinese)) or "Corona virinae19" or "Corona virinae2019" or "corona virus19" or "corona virus2019" or Coronavirinae19 or Coronavirinae2019 or coronavirus 19 or coronavirus2019 or COVID19 or COVID2019 or nCOV19 or nCOV2019 or "SARS Corona virus 2" or "SARS Coronavirus 2" or "SARS-COV-2" or "Severe Acute Respiratory Syndrome Corona virus 2" or "Severe Acute Respiratory Syndrome Coronavirus 2").ti,ab,kw. OR Coronaviridae Infections/ or Severe Acute Respiratory Syndrome/ or Coronavirus Infections/ or SARS Virus/ or Coronavirus/ AND (((sense or sensitiv* or loss or lose or lost or alter* or distort* or perception* or perceiv*) adj2 (smell* or tast* or gustation)) or anosmia* or ageusia* or dysgeusia* or parageusia* or hypogeusia* or dysosmia* or par?osmia* or hyposmia or cacosmia* or ((smell* or tast* or olfact*) adj2 (dysfunction* or disorder*)) or phantosmia or troposmia).ti,ab,kw. or olfaction disorders/ or taste disorders/ or ageusia/ or dysgeusia/ or smell/ or taste/ or taste perception/ or olfactory perception/

The search string relating to COVID-19 was adapted from a preexisting search by Ellen Aaronson at Mayo Clinic Libraries (see:

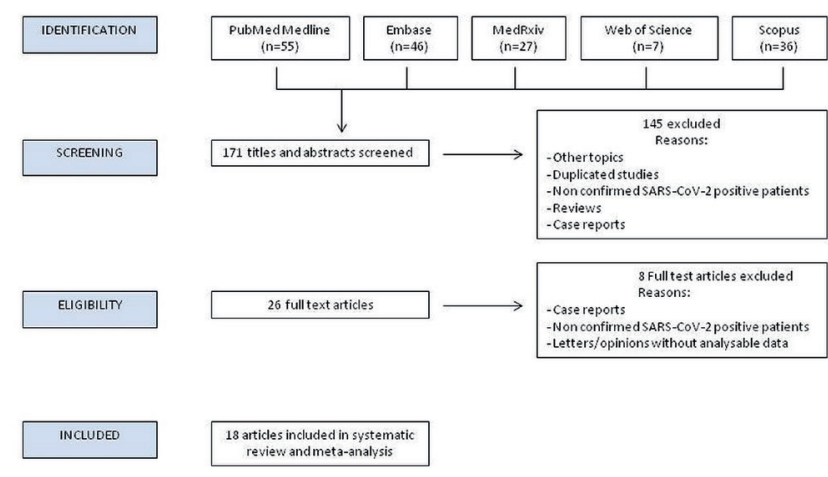

Figure 1. Flowchart of literature search strategy.

https://www.mlanet.org/page/covid-19-literature-searching)

In addition, the preprint archives MedRxiv and BioRxiv were also searched for grey literature relating to anosmia/ageusia, and COVID-19 using the following search string: (covid-19 or covid19 or coronavirus or corona virus) AND (anosmia or ageusia or olfaction or olfactory or smell or taste).

The authors also screened all articles in MedRxiv's set of COVID-19 and Otolaryngology subject preprints for relevance: https://www.medrxiv.org/collection/otolaryngology.

Two authors (DB and JP) independently screened the full texts of all the relevant articles identified against the inclusion criteria (Figure 1); a third author (PBR) settled discordances.

\section{Selection criteria}

Non-English studies were excluded. Studies containing aggregated data or duplicated data from previously published work were excluded, as were review articles, case reports, and editorials. Studies were included in the analysis if they met the following criteria: 1. the number of total patients and of those with altered sense of smell or taste were reported; 2 . symptoms were assessed in temporal proximity to the collection of swabs for PCR or hospitalization; 3. altered sense of smell or taste was self-reported; 4. confirmed SARS-CoV-2 positive patients by PCR.

\section{Data extraction and statistical analysis}

The total number of patients and those with altered sense of smell or taste were extracted from each original paper. The summary prevalence of altered sense of smell and corresponding 95\% Cl were estimated through a random intercept logistic regression model using the metaphor $\mathrm{R}$ package ${ }^{(8)}$. Statistical heterogeneity among studies was evaluated using the $R^{2}$ and $\tau^{2}$ statistics ${ }^{(9)}$. Influence analysis was performed, and pooled RR was calculated by omitting one study at a time. Publication bias was assessed through funnel plot ${ }^{(10)}$ and asymmetry was tested using a weighted linear regression of the study prevalence ${ }^{(11)}$. 
Table 1. Demographic and clinical characteristics of the study population.

\begin{tabular}{|c|c|c|c|c|c|c|c|}
\hline Author (Reference) & Country & Type of patients & Eligible & Enrolled & Women (\%) & $\begin{array}{l}\text { Median } \\
\text { age, range } \\
\text { (years) }\end{array}$ & $\begin{array}{l}\text { Symptoms' } \\
\text { evaluation }\end{array}$ \\
\hline Mao ${ }^{(5)}$ & China & Severe & - & 214 & $59 \%$ & $53( \pm 16)^{a}$ & Medical records \\
\hline Giacomelli $^{(13)}$ & Italy & Severe & 88 & 59 & $32 \%$ & $60(50-74)$ & Ad hoc questions \\
\hline Levinson ${ }^{(12)}$ & Israel & Severe & 45 & 42 & $45 \%$ & $34(15-82)$ & Ad hoc questions \\
\hline Menni ${ }^{(18)}$ & U.K. & Mild-to Moderate & - & 579 & $69 \%$ & $41( \pm 12)^{\mathrm{a}}$ & Ad hoc questions \\
\hline Spinato ${ }^{(6)}$ & Italy & Mild & 283 & 202 & $52 \%$ & $56(20-99)$ & SNOT-22 \\
\hline Moein ${ }^{(14)}$ & Iran & Severe & - & 60 & $33 \%$ & $46( \pm 12)^{\mathrm{a}}$ & Medical records \\
\hline Bénézit ${ }^{(20)}$ & France & Mild-to Moderate & - & 68 & - & - & Ad hoc questions \\
\hline Klopfenstein ${ }^{(33)}$ & France & Mild-to-Severe & - & 114 & $67 \%$ & $47( \pm 16)^{a}$ & Medical records \\
\hline Lechien ${ }^{(21)}$ & $\begin{array}{l}\text { France, Spain, } \\
\text { Belgium, Italy }\end{array}$ & Mild-to-Moderate & 1566 & 1420 & $68 \%$ & $39( \pm 12)^{\mathrm{a}}$ & Ad hoc questions \\
\hline Beltrán-Corbellini ${ }^{(15)}$ & Spain & Severe & 86 & 79 & $39 \%$ & $62( \pm 17)^{a}$ & Medical records \\
\hline Hornuss ${ }^{(16)}$ & Germany & Severe & - & 45 & $44 \%$ & $56( \pm 17)^{\mathrm{a}}$ & Medical records \\
\hline Tostmann ${ }^{(22)}$ & The Netherlands & Mild & - & 90 & $79 \%$ & - & Ad hoc questions \\
\hline Vaira ${ }^{(23)}$ & Italy & Mild-to-Moderate & - & 72 & $63 \%$ & $56(-)^{\mathrm{a}}$ & Medical records \\
\hline Wee ${ }^{(25)}$ & Singapore & Mild-to-Severe & - & 154 & - & - & Self-reported \\
\hline \multirow[t]{2}{*}{ Yan ${ }^{(24)}$} & U.S.A. & Mild-to-Moderate & - & 102 & $49 \%$ & $43(34-54)$ & Ad hoc questions \\
\hline & & Severe & - & 26 & $65 \%$ & $54(40-65)$ & Ad hoc questions \\
\hline Lechien ${ }^{(4)}$ & $\begin{array}{l}\text { France, Spain, } \\
\text { Belgium, Italy }\end{array}$ & Mild-to-Moderate & - & 417 & $63 \%$ & 37 (19-77) & sQOD-NS \\
\hline Kaye ${ }^{(19)}$ & $\begin{array}{l}\text { U.S.A. Mexico, Italy, } \\
\text { U.K., other }\end{array}$ & $\begin{array}{l}\text { Anosmic, } \\
\text { Mild-to-Moderate }\end{array}$ & 240 & 237 & $54 \%$ & $36(2-89)$ & Validated questions \\
\hline $\operatorname{Yan}^{(17)}$ & U.S.A. & Mild-to-Moderate & 102 & 59 & $49 \%$ & - & 10-point Likert scale \\
\hline
\end{tabular}

${ }^{a}$ Mean value \pm standard deviation; SNOT-22: Sino-Nasal Outcome Test 22; sQOD-NS: short version of the Questionnaire of Olfactory DisordersNegative Statements.

Analysis was stratified by severity of symptoms, i.e., studies in severely symptomatic patients versus studies in patients with mild-to-moderate symptoms.

The results of the meta-analysis were presented graphically as a forest plot, plotting the prevalence as a black square, whose size was inversely proportional to standard error. Diamonds were used to plot the summary prevalence for all studies and by study setting; their centre represents the point estimate and their extremes the $95 \% \mathrm{Cl}$. Statistical significance was set at $\mathrm{P}<0.05$ (two sided).

\section{Results}

The meta-analysis flowchart is presented in Figure 1. 18 studies met the eligibility criteria out of the 171 initially screened citations. Table 1 provides a detailed description of the clinical and demographic characteristics of all included studies. Six studies ${ }^{(5,12-16)}$ were conducted on hospitalized patients with severe symptoms, nine studies ${ }^{(4,6,17-23)}$ enrolled patients with mild-to-moderate symptoms and three studies enrolled both mild-to-moderate and severe symptomatic patients ${ }^{(19,24,25)}$.
Two studies ${ }^{(21,24)}$ included all cases reported in two previously published papers ${ }^{(4,17)}$ enriching them with further cases; as the first published papers provided information on the timing of the onset of smell and taste impairment not present in the casesenriched papers, they were considered only for this purpose. Another study was conducted on anosmic patients only and was considered only in the evaluation of the timing of the onset of smell impairment ${ }^{(19)}$. Thus, out of 3563 single cases included in the meta-analysis, 3326 have been considered for prevalence calculation. Three studies ${ }^{(4,6,17)}$ assessed alteration of sense of smell or taste through a validated questionnaire whereas ad hoc questions where used in other studies.

Overall, the prevalence of alteration of sense of smell or taste was $47 \%$ (95\% Cl: 36\%-59\%); great heterogeneity was observed, with prevalence ranging from $11 \%$ to $84 \%$ (Figure 2). Funnel plot (Supplementary Figure 1) reflects the potential for publication bias, thus asymmetry was not statistically significant $(p=0.08)$. Notably, great heterogeneity emerged with respect to patients' clinical condition (Figure 3): among 525 severely symptomatic patients, only 139 subjects reported alteration of sense of smell 


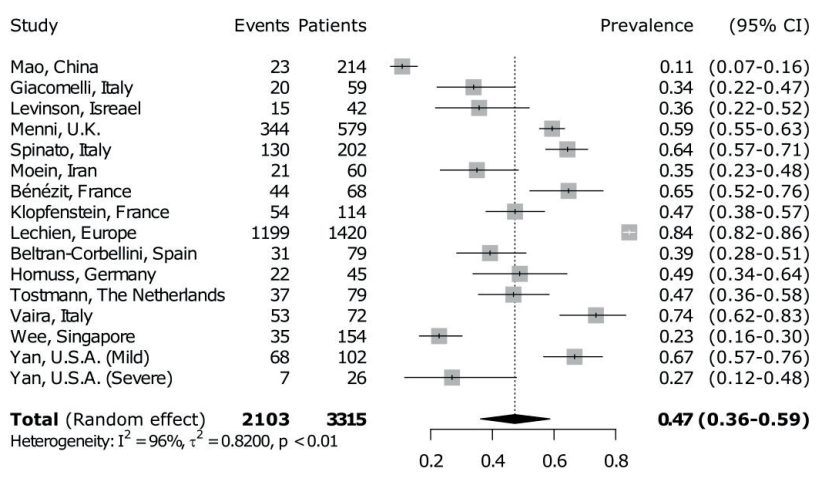

Figure 2. Forest plot of the prevalence of alteration of the sense of smell or taste in 3315 patients. Each study prevalence is displayed through as a black square, whose size was inversely proportional to standard error; horizontal bar represents $95 \%$ confidence interval. Diamond represents the summary prevalence for all studies.

or taste, leading to a pooled symptom's prevalence of 31\% (95\% $\mathrm{Cl}: 22 \%-42 \%)$. The prevalence was much higher among patients with mild-to-moderate symptoms (67\%; 95\% Cl: 58\%-75\%); the study by Lechien ${ }^{(21)}$ was the most influent, which exclusion lowered the pooled estimate to $62 \%$ (95\% Cl: 57\%-68\%; data not shown).

Four studies $(4,6,1,19)$ in mildly-to-moderately symptomatic patients reported information on timing of onset of alteration of sense of smell or taste with respect to other symptoms. Among patients with alteration of sense of smell or taste, this symptom preceded the others in $20 \%$ (95\% Cl: $13 \%-29 \%$ ) of cases, whereas it was concomitant in 28\% (95\% Cl: 22\%-36\%). Alteration of sense of smell or taste occurred after other symptoms in 54\% (95\% Cl: $37 \%-70 \%)$ of symptomatic cases. Prevalence do not add up to $100 \%$ since not all studies reported complete information on timing of symptoms' onset (Figure 4).

\section{Discussion}

The results from this meta-analysis suggest a robust association between altered sense of smell or taste and confirmed SARSCoV-2 infection. The overall prevalence of smell or taste impairment in patients with COVID-19 was $47 \%$, which rises up to $67 \%$ in patients with mild-to-moderate symptoms; $20 \%$ of all patients reported it as an isolated or as the first presenting symptom.

The presence of the loss of smell is not surprising, as many human respiratory viral strains, including coronavirus have been observed to cause post-viral anosmia ${ }^{(26)}$. Human coronaviruses have been indeed demonstrated to invade the central nervous system through the olfactory neuroepithelium and propagate from within the olfactory bulb (2). Furthermore, nasal epithelial cells were observed to display the highest expression of the

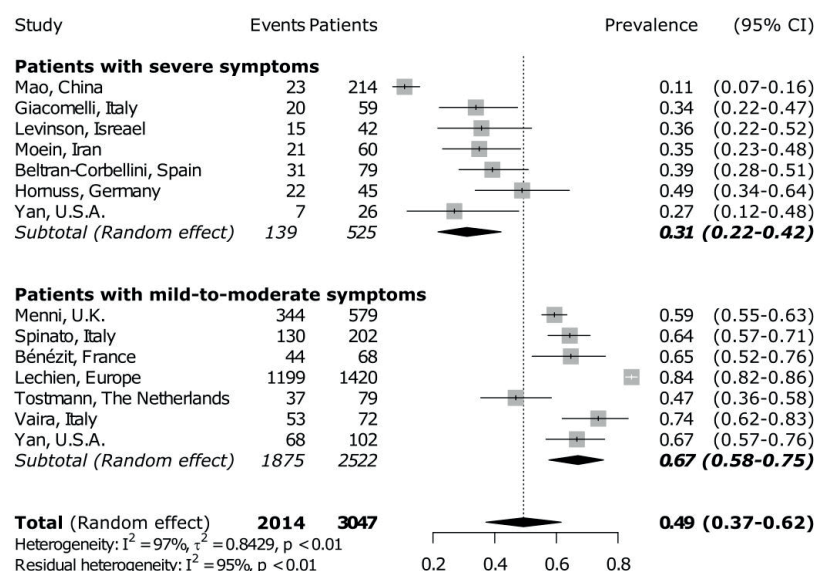

Figure 3. Forest plot of the prevalence of alteration of the sense of smell according to patients' clinical condition (severe versus mild-to-moderate symptoms). Each study prevalence is displayed through as a black square, whose size was inversely proportional to standard error; horizontal bar represents $95 \%$ confidence interval. Diamonds represent the summary prevalences.

SARS- CoV-2 receptor, ACE-2, in the respiratory tree making the nasal epithelium a likely target of viral infection ${ }^{(27)}$.

First clues of an association between an altered sense of smell or taste and the SARS-CoV-2 infection came from anecdotal reports describing an increase in reports of these symptoms occurring at the outset of the COVID-19, such that the British Association of Otorhinolaryngologists ${ }^{(28)}$ and the American Academy of Otolaryngology-Head and Neck Surgery ${ }^{(29)}$ issued warnings to consider the diagnosis of SARS-CoV-2 with isolated lost or altered sense of smell or taste. At the end of March, Gane et al. documented the first case report of isolated sudden onset anosmia in a confirmed case of SARS-CoV-2 infection as well as a small case series highlighting the increase in referrals for sudden onset anosmia at the beginning of the spread of SARS-CoV- 2 infection in the United Kingdom ${ }^{(30)}$. Other indirect evidence came from a non-peer reviewed manuscript, made available on a preprint server reporting a linear correlation between prevalence of COVID-19 and olfactory impairment in Iran ${ }^{(31)}$. Since these early reports there has been a rapid evolution in the peer reviewed literature.

This meta-analysis focused on self-reported impairment of sense of smell or taste, to provide robust data on symptoms that can be assessed during patient's anamnesis without the need of specialist tests. These results are however further supported by the first study also assessing olfactory dysfunction through objective testing ${ }^{(14)}$. An Iranian study, conducted on 60 SARS-CoV2-positive inpatients and age matched controls, assessed the olfactory status through a validated 40 -odorant test, reporting 


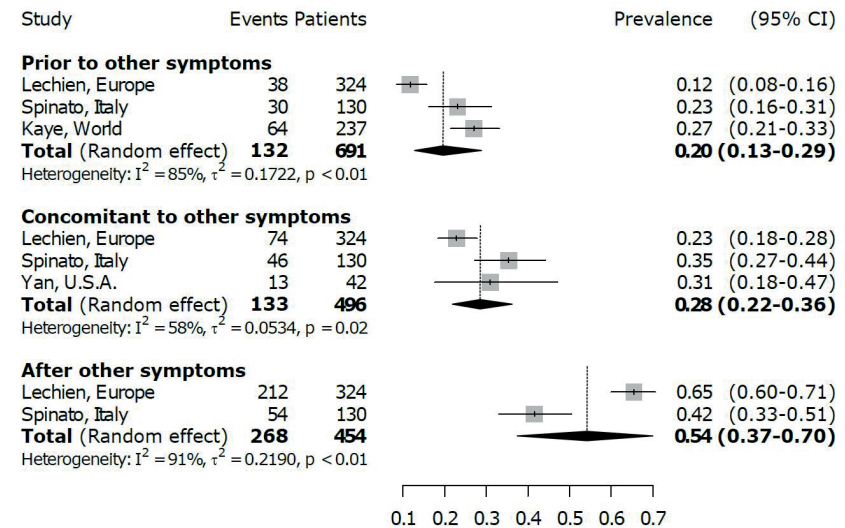

Figure 4. Forest plot of the timing of onset of alteration of sense of smell or taste with respect to other symptoms. Each study prevalence is displayed through as a black square, whose size was inversely proportional to standard error; horizontal bar represents $95 \%$ confidence interval. Diamonds represent the summary prevalences.

a prevalence of smell dysfunction of $98 \%$, with $58 \%$ rated as anosmic, while only $18 \%$ of controls were found to have smell dysfunction, of which all were mild with none rated as anosmic. It is interesting that only $35 \%$ of patients self-reported loss of smell and taste, suggesting that self-rating of sense of smell or taste will likely underestimate the true prevalence of olfactory dysfunction in COVID-19 patients.

Through a systematic review of the literature we selected paper sreporting the prevalence of self-reported smell or taste impairment in confirmed cases of SARS-CoV-2 infection. The following observations can be highlighted from this systematic review and meta-analysis. First, we found that the prevalence of an altered sense of smell or taste is highly dependent on the setting. While in patients with severe symptoms the overall prevalence was $31 \%$, it raised to $67 \%$ in mild-to-moderate symptomatic home-isolated patients. Possibly, in patients with more severe COVID-19 symptoms, symptoms around olfaction may not have been elicited and recorded, or simply neglected in the setting of more severe respiratory symptoms. In addition, some authors speculate that paucisymptomatic patients may be affected by nasal-centric viral spread, whereas patients requiring hospitalization may be experiencing a more pulmonary-centric viral infection leading to increased rate of respiratory failure and need for hospitalization ${ }^{(17)}$. In addition, the loss of smell or taste can precede (20\%) or be concomitant to other presentation symptoms (28\%). Thus, smell or taste impairment may be the first symptom of SARS-CoV-2 infection in a substantial proportion of cases. Consequently, current guidelines should recognize isolated sudden onset of the loss of smell or taste as a criterion for self-isolation. It has been shown, indeed, that undocumented asymptomatic or mildly symptomatic infections substantially facilitate the rapid dissemination of SARS-CoV-2 ${ }^{(32)}$.

This meta-analysis has its limitations in the nature of the paper analysed: symptoms were self-reported, based on cross-sectional surveys or retrospective case series, and thus may suffer from sub-optimal sensitivity. These types of studies are in fact intrinsically predisposed to selection bias. Some of the studies involved small number of subjects; studies based on crosssectional survey of defined patient cohorts had a response rate ranging from $58 \%{ }^{(17)}$ to $93 \%{ }^{(12)}$. Other studies relied on voluntary completion of a questionnaire or use of an online symptom tracker; the respondents may therefore have an age and gender bias related to the tendency to engage with such studies. Publication bias is also possible, as suggested by funnel plot inspection: this is plausible, considering the rapid spread of the epidemic and the urgency to disseminate scientific data.

More specifically, the two studies by Lechien et al. includes a mixed population of respondents from different settings and incorporates a high proportion of volunteer health-care workers, thus suffering by a potential recruitment bias that, as stated by the authors, may have led to an overestimation of the loss of sense of smell and taste in that case series ${ }^{(4,21)}[4,21]$; the study by Yan et al. had a low response rate to the survey so having the risk the participants were a non-representative sample (17); both the retrospective observational case series by Mao et al. ${ }^{(5)}$ and Klopfenstein et al. ${ }^{(33)}$ might have underestimated the burden of the loss of sense of smell and taste in their series as they proceeded to extract data from medical records, therefore certain mild symptoms, including smell and taste impairment, might not be captured in all patients. Finally, as this is a rapidly evolving area, we also included pre-print literature. However, we did not find severe methodological problems in the not yet peer-reviewed papers we included and by excluding these papers we would have lost an important source of information.

We have not attempted to differentiate between self-reported loss of smell or loss of taste. It has been shown that patients will often report perceived loss of taste in cases of olfactory dysfunction due to impaired flavour perception; a high incidence in flavour disturbance being reported by patients with sudden post-viral olfactory loss ${ }^{(34)}$, despite normal gustatory function. Furthermore, focused items on taste perception (relating to salt, sweet, bitter or sour tastes) may not be reliable in differentiating disturbances in the perception of taste and flavour ${ }^{(35)}$. We therefore feel that in order to be of greatest practical application, self-rated disturbance of smell or taste should be considered in combination for the purposes of case-identification.

\section{Conclusion}

In conclusion, this analysis has summarised the current evidence base, overcoming the limitations caused by small sample size. 
Based on this meta-analysis, we therefore consider it reasonable to recommend self-isolation for patients complaining of smell or taste impairment, pending execution and the result of formal testing where capacity exists, during COVID-19 pandemic. Sudden onset of these symptoms may be used for tracking and as a screening tool for this disease. There is also a need for raising awareness and educating patients: that mildly symptomatic patients with only the loss of sense of smell or taste may indeed become a source of SARS-CoV-2 dissemination if they continue to work or socialize. It is also quite important to educate healthcare professionals, in particular general practitioners and otolaryngologists, to the fact that an impairment of the sense of smell or taste could be a suspicious symptom of SARS-CoV-2 infection, thus, to prevent spread of disease, they should avoid nasal endoscopy, and employ full personal protective equipment to reduce transmission. When the peak of the pandemic will cease, bringing with it easing of the protection measures and social restrictions, recognizing these symptoms, will become even more significant in order to promptly identify patients at risk for SARS-CoV-2 infection and virus dissemination. Finally, we propose inclusion of anosmia in the World Health Organization and other relevant regulatory body's lists of recognized symptoms of SARS-CoV-2.

\section{Acknowledgements}

None

\section{Authorship contribution}

PBR has led on the concept and written the initial draft with the help of DB, who conducted the systematic review. VP searched for the literature. JP performed the statistical analysis. $\mathrm{CH}, \mathrm{RO}$, JP and GT helped writing the manuscript. All authors reviewed final draft.

\section{Conflict of interest}

None

\section{References}

1. Huang C, Wang Y, Li X, Ren L, Zhao J, Hu $Y$, et al. Clinical features of patients infected with 2019 novel coronavirus in Wuhan, China. Lancet Lond Engl 2020;395:497-506.

2. Dubé $M$, Le Coupanec A, Wong AHM, Rini JM, Desforges M, Talbot PJ. Axonal Transport Enables Neuron-to-Neuron Propagation of Human Coronavirus OC43. J Virol 2018;92.

3. Fodoulian L, Tuberosa J, Rossier D, Landis BN, Carleton A, Rodriguez I. SARS-CoV-2 receptor and entry genes are expressed by sustentacular cells in the human olfactory neuroepithelium. BioRxiv 2020:2020.03.31.013268.

4. Lechien JR, Chiesa-Estomba CM, De Siati DR, Horoi M, Le Bon SD, Rodriguez A, et al. Olfactory and gustatory dysfunctions as a clinical presentation of mild-to-moderate forms of the coronavirus disease (COVID19): a multicenter European study. Eur Arch Otorhinolaryngol. 2020 Apr 6:1-11.

5. Mao L, Jin H, Wang M, Hu Y, Chen $S$, He $Q$, et al. Neurologic Manifestations of Hospitalized Patients With Coronavirus Disease 2019 in Wuhan, China. JAMA Neurol. 2020 Apr 10;77(6):1-9.

6. Spinato G, Fabbris C, Polesel J, Cazzador D, Borsetto D, Hopkins C, et al. Alterations in Smell or Taste in Mildly Symptomatic Outpatients With SARS-CoV-2 Infection. JAMA. 2020 Apr 22;323(20):2089-90.

7. Moher D, Shamseer L, Clarke M, Ghersi $D$, Liberati A, Petticrew $M$, et al. Preferred reporting items for systematic review and meta-analysis protocols (PRISMA-P) 2015 statement. Syst Rev 2015;4:1.

8. Stijnen T, Hamza TH, Ozdemir P. Random effects meta-analysis of event outcome in the framework of the generalized linear mixed model with applications in sparse data. Stat Med 2010;29:3046-67.

9. Normand SL. Meta-analysis: formulating, evaluating, combining, and reporting. Stat Med 1999;18:321-59.

10. Sterne JA, Egger M. Funnel plots for detecting bias in meta-analysis: guidelines on choice of axis. J Clin Epidemiol 2001;54:1046-55

11. Peters JL, Sutton AJ, Jones DR, Abrams KR, Rushton L. Comparison of two methods to detect publication bias in meta-analysis. JAMA 2006;295:676-80.

12. Levinson R, Elbaz M, Ben-Ami R, Shasha D, Levinson $T$, Choshen $G$, et al. Anosmia and dysgeusia in patients with mild SARS-CoV-2 infection. MedRxiv 2020:2020.04.11.20055483.

13. Giacomelli A, Pezzati L, Conti F, Bernacchia $D$, Siano M, Oreni L, et al. Self-reported Olfactory and Taste Disorders in Patients With Severe Acute Respiratory Coronavirus 2 Infection: A Cross-sectional Study. Clin Infect Dis. 2020 Jul 28;71(15):889-890.

14. Moein ST, Hashemian SMR, Mansourafshar B, Khorram-Tousi A, Tabarsi P, Doty RL. Smell dysfunction: a biomarker for COVID-19. Int Forum Allergy Rhinol. 2020 Aug;10(8):953954.

15. Beltrán-Corbellini Á, Chico-García JL, Martínez-Poles J, Rodríguez-Jorge F, NateraVillalba E, Gómez-Corral J, et al. Acute-onset smell and taste disorders in the context of Covid-19: a pilot multicenter PCR-based case-control study. Eur J Neurol. 2020 May 25:10.1111/ene.14359.

16. Hornuss D, Lange B, Schroeter N, Rieg S, Kern WV, Wagner D. Anosmia in COVID-19 patients. MedRxiv 2020:2020.04.28.20083311.

17. Yan CH, Faraji F, Prajapati DP, Boone CE, DeConde AS. Association of chemosensory dysfunction and Covid-19 in patients presenting with influenza-like symptoms. Int Forum Allergy Rhinol. 2020 Jul;10(7):806813.

18. Menni C, Valdes A, Freydin MB, Ganesh S, Moustafa JE-S, Visconti A, et al. Loss of smell and taste in combination with other symptoms is a strong predictor of COVID-19 infection. MedRxiv 2020:2020.04.05.20048421.

19. Kaye R, Chang CWD, Kazahaya K, Brereton J, Denneny JC. COVID-19 Anosmia Reporting Tool: Initial Findings. Otolaryngol Head Neck Surg. 2020 Jul;163(1):132-134.

20. Bénézit F, Turnier PL, Declerck C, Paillé C, Revest M, Dubée $V$, et al. Utility of hyposmia and hypogeusia for the diagnosis of COVID19. Lancet Infect Dis. 2020 Apr 15;S14733099(20)30297-8,

21. Lechien JR, Chiesa-Estomba CM, Place S, Laethem YV, Cabaraux P, Mat Q, et al. Clinical and Epidemiological Characteristics of 1,420 European Patients with mild-tomoderate Coronavirus Disease 2019. J Intern Med. 2020 Sep;288(3):335-344.

22. Tostmann A, Bradley J, Bousema T, Yiek W-K, Holwerda M, Bleeker-Rovers C, et al. Strong associations and moderate predictive value of early symptoms for SARS-CoV-2 test positivity among healthcare workers, the Netherlands, March 2020. Eurosurveillance 2020;25:2000508.

23. Vaira LA, Deiana $G$, Fois $A G$, Pirina $P$, Madeddu G, De Vito A, et al. Objective evaluation of anosmia and ageusia in COVID-19 patients: Single-center experience on 72 cases. Head Neck 2020 Jun;42(6):1252-1258.

24. Yan CH, Faraji F, Prajapati DP, Ostrander BT, DeConde AS. Self-reported olfactory loss associates with outpatient clinical course in Covid-19. Int Forum Allergy Rhinol. 2020 
Jul; 10(7):821-831.

25. Wee LE, Chan YFZ, Teo NWY, Cherng BPZ, Thien SY, Wong HM, et al. The role of selfreported olfactory and gustatory dysfunction as a screening criterion for suspected COVID-19. Eur Arch Otorhinolaryngol. 2020 Aug;277(8):2389-2390.

26. Suzuki M, Saito K, Min W-P, Vladau C, Toida $\mathrm{K}$, Itoh $\mathrm{H}$, et al. Identification of viruses in patients with postviral olfactory dysfunction. Laryngoscope 2007;117:272-7.

27. Sungnak W, Huang N, Bécavin C, Berg M, Network HLB. SARS-CoV-2 Entry Genes Are Most Highly Expressed in Nasal Goblet and Ciliated Cells within Human Airways. Nat Med. 2020 May;26(5):681-687.

28. COVID-19 n.d. https://www.entuk.org/ covid-19 (accessed April 22, 2020).

29. AAO-HNS: Anosmia, Hyposmia, and Dysgeusia Symptoms of Coronavirus Disease, American Academy of Otolaryngology-Head and Neck Surgery n.d. https://www.entnet.org/content/aaohns-anosmia-hyposmia-and-dysgeusiasymptoms-coronavirus-disease (accessed March 28, 2020).

30. Gane SB, Kelly C, Hopkins C. Isolated sudden onset anosmia in COVID-19 infection. A novel syndrome? Rhinology 2020 Jun 1;58(3):299-301.

31. Bagheri SHR, Asghari AM, Farhadi $M$, Shamshiri AR, Kabir A, Kamrava SK, et al. Coincidence of COVID-19 epidemic and olfactory dysfunction outbreak. MedRxiv 2020:2020.03.23.20041889.

32. Li R, Pei S, Chen B, Song Y, Zhang T, Yang W, et al. Substantial undocumented infection facilitates the rapid dissemination of novel coronavirus (SARS-CoV2). Science 2020 May 1;368(6490):489-493.

33. Klopfenstein T, Kadiane-Oussou NJ, Toko L, Royer P-Y, Lepiller Q, Gendrin V, et al. Features of anosmia in COVID-19. Med Mal Infect. 2020 Aug;50(5):436-439.
34. Fujii M, Fukazawa K, Hashimoto Y, Takayasu S, Umemoto M, Negoro A, et al. Clinical study of flavor disturbance. Acta OtoLaryngol Suppl 2004:109-12.

35. Soter A, Kim J, Jackman A, Tourbier I, Kaul $A$, Doty RL. Accuracy of self-report in detecting taste dysfunction. Laryngoscope 2008;118:611-7.

\section{Daniele Borsetto}

Guy's Hospital

London SE1 9RT

United Kingdom

Tel: +447853 222040

E-mail:daniele.borsetto@gmail.com

\section{SUPPLEMENTARY DATA}

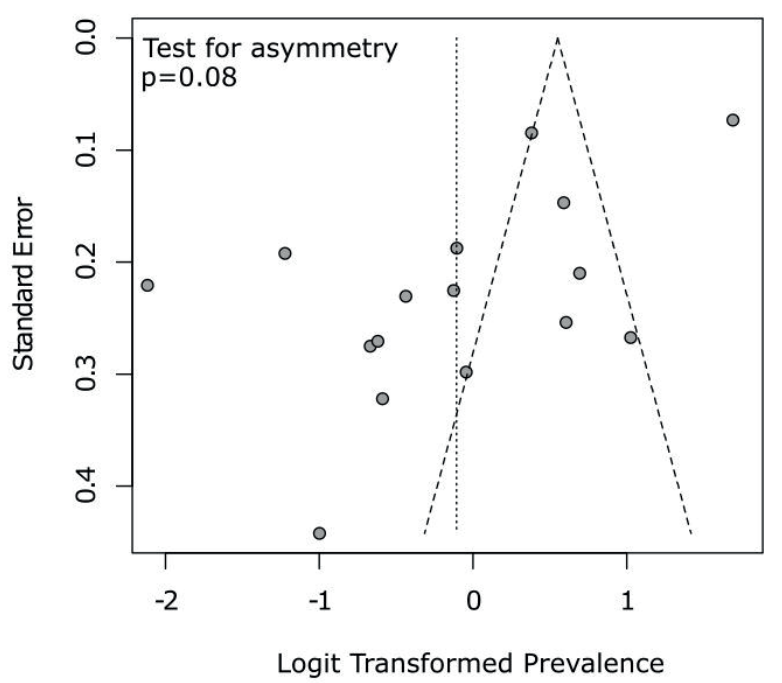

Supplementary Figure 1. Funnel plot investigating potential publication bias. Asymmetry was tested using a weighted linear regression of the study prevalence. 